\title{
Genotipificación de Plasmodium falciparum por PCR múltiple por medio de los genes msp1, msp2 y glurp, en cuatro localidades de Colombia
}

\author{
Sandra Milena Barrera1', Manuel Alberto Pérez², Angélica Knudson², Rubén Santiago Nicholls², \\ Ángela Patricia Guerra² \\ 1 Grupo de Bioquímica y Biología Celular, Instituto Nacional de Salud, Bogotá, D.C., Colombia \\ ${ }^{2}$ Grupo de Parasitología, Instituto Nacional de Salud, Bogotá, D.C., Colombia
}

Introducción. La diversidad genética de Plasmodium falciparum constituye un obstáculo para el éxito de la terapia antipalúdica, pues le permite al parásito evadir la respuesta inmunitaria del huésped, lo cual genera cambios en su composición antigénica y resistencia a los medicamentos antipalúdicos.

Objetivo. Estudiar la diversidad genética de $P$. falciparum procedente de cuatro localidades colombianas mediante el análisis de los genes polimórficos $m s p 1, m s p 2$ y glurp.

Materiales y métodos. Se genotipificaron 81 muestras mediante PCR múltiple de pacientes infectados con P. falciparum, procedentes de Tierralta (Córdoba), Inírida (Guainía), La Carpa (Guaviare) y Casuarito (Vichada).

Resultados. Para el gen msp1 se detectó MAD20 en todas las muestras analizadas. Para el gen msp2 se halló con mayor frecuencia la familia alélica IC (96,3\%) comparada con FC (4,9\%). En ambas familias se evidenció polimorfismo de tamaño, y se encontraron bandas en un rango entre 467 y 513 pares de bases (pb) para IC y entre 286 y 300 pb para FC. Para el gen glurp se detectaron diferentes tamaños de productos de PCR, los cuales se agruparon en cinco genotipos: I (600-699 pb) 2,5\%, II (700-799 pb) 19,8\%, III (800-899 pb) 72,8\%, IV (900-999 pb) 1,2\% y V (1.000-1.099 pb) 3,7\%.

Conclusiones. Nuestros resultados demuestran que el marcador molecular $m s p 1$ no proporciona información útil para diferenciar las poblaciones parasitarias de $P$. falciparum. El gen $m s p 2$ es apropiado para evaluar la diversidad genética, pero requiere ensayos más finos que permitan diferenciar claramente el polimorfismo de tamaño en las dos familias. Los resultados obtenidos con el gen glurp evidenciaron una gran diversidad genética en las poblaciones de $P$. falciparum que circulan en el país y sugieren que este gen puede ser útil para diferenciar nuevas infecciones de infecciones recurrentes o recrudecimientos.

Palabras clave: Plasmodium falciparum, malaria, reacción en cadena de la polimerasa, variación genética, Colombia.

Genotypic survery of Plasmodium falciparum based on the msp1, msp2 and glurp genes by multiplex PCR

Introduction. The genetic diversity of Plasmodium falciparum has been one of the major obstacles for the success of anti-malaria drug therapy. It provides the parasite an ability to evade the host's immune response by generating changes in its antigenic composition and resistance to antimalarial drugs.

Objective. The genetic diversity of P.falciparum was characterized in 4 Colombian localities through the analysis of polymorphic genes.

Materials and methods. Eighty-one samples were obtained from patients with uncomplicated $P$. falciparum malaria and screened for polymorphic variants of $m s p 1, m s p 2$ (merozoite surface proteins) and glurp (glutamate-rich protein) with a multiplex PCR assay. The geographic regions sampled were Tierralta (Córdoba), in northwestern Colombia and in the Orinoco river watershed of eastern Colombia-Inírida (Guainía), La Carpa (Guaviare), and Casuarito (Vichada).

Results. The MAD20 variant was detected in all samples analyzed for the $m s p 1$ gene. For the $m s p 2$ gene, the IC allelic family was found in $96.3 \%$ of the samples as compared to $4.9 \%$ of the samples with the FC family. Both families showed size polymorphism with bands between 467 and 513 basepairs (bp) for IC and 286 and 300 bp for FC. PCR products of differing sizes were detected for the glurp gene and grouped into 5 size classes: I (600-699 bp) 2.5\%, II (700-799bp) 19.8\%, III (800-899 bp) $72.8 \%$, IV (900-999 bp) $1.2 \%$ and V (1000-1099 bp) 3.7\%.

Conclusions. The msp1 molecular marker did not provide information for differentiating $P$. falciparum parasite populations. The msp 2 gene was more suitable for studying the genetic diversity, however, further studies are required to identify polymorphisms within the two allelic families. The glurp gene 
showed a great genetic diversity of circulating $P$. falciparum populations, and suggested that this gene may be useful for distinguishing between recrudescence and reinfection.

Key words: Plasmodium falciparum, malaria, polymerase chain reaction, genetic variation, Colombia.

La malaria continúa siendo uno de los principales problemas de salud pública en el mundo (1). En Colombia, en el año 2007 se reportaron 109.277 casos distribuidos por especie entre Plasmodium falciparum, 29.997 (27,45\%), P. vivax, 77.987 $(71,37 \%)$, P. malarie, $18(0,01 \%)$ e infecciones mixtas, 1.275 (1,17\%) (2). Plasmodium falciparum es la especie causante de las formas graves de la enfermedad y de la mayoría de las muertes.

La diversidad genética y la multiplicidad de Plasmodium spp. son en parte responsables del éxito biológico de este parásito y del fracaso en las medidas empleadas para el control de la malaria, ya que le confieren la capacidad de evadir la respuesta inmunitaria del huésped y desarrollar resistencia a los medicamentos antipalúdicos $(3,4)$. La gran capacidad de recombinación genética del parásito le permite generar nuevas combinaciones de su material genético y la producción de diversas proteínas para evadir la respuesta inmunitaria del huésped vertebrado, incrementando las tasas de variabilidad en la composición antigénica de las formas infectantes, aspecto que se asocia con dificultades en el desarrollo de vacunas (4) y se convierte en uno de los retos por afrontar más importantes para el desarrollo de estrategias terapéuticas efectivas (3).

La diversidad genética en $P$. falciparum se ha analizado mediante genes polimórficos que codifican para proteínas de superficie, entre los cuales se encuentran msp1, msp2 y glurp (5-7). Estos genes se presentan en copia única durante la fase asexual del parásito, muestran grandes diferencias entre las poblaciones naturales de $P$. falciparum y presentan polimorfismos que varían de acuerdo con las características epidemiológicas de cada región (8-11). El gen glurp está constituido por una región conservada no repetida $(\mathrm{RO})$ y dos regiones repetidas ( $R 1$ y $R 2$ ). La región $R 2$ es de aproximadamente $60 \mathrm{pb}$ y varía entre las diferentes cepas de parásitos $(9,12-14)$. El gen msp1 está

\footnotetext{
Correspondencia:

Ángela Patricia Guerra, Grupo de Bioquímica y Biología Celular, Instituto Nacional de Salud, Avenida calle 26 № 51-20, zona 6, CAN, Bogotá, D.C., Colombia.

Teléfono: (+571) 220 7700, extensiones 1348 y 1349

aguerra@ins.gov.co

Recibido: 21/09/09; aceptado:03/07/10
}

constituido por 17 bloques, de los cuales siete son muy variables $(4,15,16)$. El gen $m s p 2$ contiene una secuencia polimórfica denominada bloque central o 3; los extremos de este bloque están constituidos por secuencias no repetidas conservadas que clasifican al gen en dos grandes familias de alelos, denominadas 3D7/IC y FC27 (FC) $(5,6,17)$.

Los estudios de diversidad genética en $P$. falciparum realizados en Colombia han empleado principalmente los genes msp1 y msp2. En 1991, Snewin reportó la familia $\mathrm{RO} 33$ como el alelo predominante en msp1 (18). Sin embargo, todos los trabajos realizados posteriormente mostraron un predominio de la familia MAD20 con algunas diferencias muy sutiles en tamaño (12,19-21), mientras que para msp2 se observó una frecuencia mayor de $90 \%$ para la familia del alelo IC con respecto a la FC $(12,19)$. Son pocos los trabajos donde se evalúan simultáneamente los tres marcadores (msp1, msp2 y glurp); sin embargo, en ellos se ha podido mostrar el mayor aporte del gen glurp en cuanto a diversidad de los parásitos $(22,23)$.

En Suramérica se han realizado algunas investigaciones utilizando el gen glurp. En un trabajo llevado a cabo en Perú se encontraron abundantes polimorfismos de tamaño, resultado que permitió definir ocho genotipos distintos (13).

Teniendo en cuenta que en Colombia los marcadores msp1 y msp2 no han permitido diferenciar las poblaciones circulantes de $P$. falciparum, el escaso número de estudios con el gen glurp en nuestro país y los resultados de Perú, decidimos incluir este marcador en el presente trabajo para establecer su utilidad en estudios de diversidad genética, comparando el polimorfismo presente en estos tres genes, en muestras de cuatro localidades ubicadas en zonas con distinto grado de endemia de malaria en Colombia. En este estudio se analizó la región $\mathrm{R} 2$ del gen glurp, el bloque 2 del gen msp1 constituido por tres familias alélicas (K1, MAD20 Y RO33), y el bloque 3 del gen msp2 (4-6,12-17).

\section{Materiales y métodos}

\section{Sitios de estudio}

El estudio se efectuó con muestras de pacientes con malaria no complicada por $P$. falciparum, 
procedentes de cuatro localidades colombianas situadas en diferentes regiones del país (UrabáBajo Cauca-Alto Sinú, Orinoquia y Amazonia), con distintos grados de endemia para malaria.

De las 81 muestras, 59 fueron recolectadas en el municipio de Tierralta, en el departamento de Córdoba, de mayo a noviembre de 2006, como parte de un estudio de eficacia in vivo. Ésta es una de las zonas de más alta incidencia y transmisión de malaria en el país, con un índice parasitario anual mayor de 10 por 1.000 habitantes y considerada zona de alto riesgo epidemiológico $(2,24)$. Por otro lado, se obtuvieron dos muestras de la localidad de Inírida, en el departamento de Guainía, una recolectada en abril y la otra en julio de 2007; 10 muestras de La Carpa, corregimiento de San José del Guaviare (Guaviare) y 10 de Casuarito, corregimiento de Puerto Carreño (Vichada), recolectadas en los años 2003 y 2004, respectivamente.

\section{Recolección de las muestras}

Se recolectó sangre de pacientes infectados con $P$. falciparum, la cual fue impregnada en círculos de papel filtro Whatman $3 \mathrm{M}$ de $1 \mathrm{~cm}$ de diámetro, antes de la administración del tratamiento (día 0). Además, se recolectó una muestra el día que se presentó la falla al tratamiento (precoz: día 2, y tardía: día 28), de los tres pacientes de Tierralta que presentaron fracaso terapéutico (24). Estas muestras también se recolectaron en papel filtro y todas se almacenaron a temperatura ambiente en bolsa plástica individual y protegida de la luz solar hasta su llegada al laboratorio.

\section{Extracción del ADN del parásito}

La técnica empleada fue Saponina/Chelex. Consiste en cortar un pedazo de la muestra obtenida en papel filtro y adicionar $1 \mathrm{ml}$ de saponina al $0,5 \%$, incubando durante toda la noche a $4{ }^{\circ} \mathrm{C}$. Al día siguiente se descarta la saponina y se agrega $1 \mathrm{ml}$ de solución salina amortiguada con fosfatos (PBS) $1 \mathrm{X}$ por 30 minutos a $4{ }^{\circ} \mathrm{C}$; en un tubo adicional se agregan $200 \mu \mathrm{l}$ de una solución de Chelex al 5\% y se introduce en un bloque de calentamiento a 99 ${ }^{\circ} \mathrm{C}$; después de tres minutos se agrega el papel con la muestra y se agita por 10 minutos; luego se centrifuga el microtubo por tres minutos a 13.000 rpm y se retira el sobrenadante (ADN), evitando tomar la resina. EI ADN se distribuye en alícuotas de $30 \mu \mathrm{l}$ y se almacena a $-20^{\circ} \mathrm{C}$.

\section{Genotipificación mediante PCR múltiple}

Se utilizó el protocolo descrito por Snounou, et al. (9) y Viriyakosol, et al. (25) con algunas modificaciones. Se amplificaron los genes msp1, msp2 y glurp en una sola reacción de PCR usando las siguientes condiciones: solución tampón $1 \mathrm{X}$, $\mathrm{MgCl}_{2} 1,5 \mathrm{mM}$, dNTPs $125 \mu \mathrm{M}$, iniciadores msp1, msp2 y glurp $125 \mathrm{nM}$, Taq polimerasa 0,4 U/PCR y $1,5 \mu \mathrm{l}$ de ADN, con un volumen final de $50 \mu \mathrm{l}$ por tubo. Los iniciadores para amplificar el gen msp1 fueron M1-OF y M1-OR, para msp2, M2-OF y M2-OR, y para glurp, G-OF y G-OR (cuadro 1). El programa de amplificación usado en la PCR

Cuadro 1. Secuencias de los oligonucleótidos utilizadas como iniciadores en la reacción en cadena de polimerasa para la amplificación de los genes msp1, msp2 y glurp.

\begin{tabular}{|c|c|c|c|}
\hline Gen & Amplicón & Iniciador & Secuencia $5^{\prime}-3^{\prime}$ \\
\hline \multirow[t]{7}{*}{ msp1 } & Bloque 2 & $\begin{array}{l}\text { M1-OF } \\
\text { M1-OR }\end{array}$ & $\begin{array}{l}\text { CTA GAA GCT TTA GAA GAT GCA GTA TTG } \\
\text { CTT AAA TAG TAT TCT AAT TCA AGT GGA TCA }\end{array}$ \\
\hline & $\mathrm{K} 1$ & M1-2KF & AAA TGA AGA AGA AAT TAC TAC AAA AGG TGC \\
\hline & & $\mathrm{M} 1-2 \mathrm{KR}$ & GCT TGC ATC AGC TGG AGG GCT TGC ACC AGA \\
\hline & MAD20 & M1-2MF & AAA TGA AGG AAC AAG TGG AAC AGC TGT TAC \\
\hline & & M1-2MR & ATC TGA AGG ATT TGT ACG TCT TGA ATT ACC \\
\hline & $\mathrm{RO} 33$ & M1-2RF & TAA AGG ATG GAG CAA ATA CTC AAG TTG TTG \\
\hline & & M1-2RR & CAT CTG AAG GAT TTG CAG CAC CTG GAG ATC \\
\hline \multirow[t]{6}{*}{ msp2 } & Bloque 3 & M2-OF & ATG AAG GTA ATT AAA ACA TTG TCT ATT ATA \\
\hline & & M2-OR & CTT TGT TAC CAT CGG TAC ATT CTT \\
\hline & IC & M2-FCF & AAT ACT AAG AGT GTA GGT GCA A/GAT GCT CCA \\
\hline & & M2-FCR & TTT TAT TTG GTG CAT TGC CAG AAC TTG AAC \\
\hline & $\mathrm{FC}$ & M2-ICF & AGA AGT ATG GCA GAA AGT AAG/T CCT C/TCT ACT \\
\hline & & M2-ICR & GAT TGT AAT TCG GGG GAT TCA GTT TGT TCG \\
\hline \multirow[t]{4}{*}{ Glurp } & Región RII & G-OF & TGA AAT TGA AGA TGT TCA CAC TGA AC \\
\hline & & G-OR & GTG GAA TTG CTT TTT CTT CAA CAC TAA \\
\hline & Región RII & G-NF & TGT TCA CAC TGA ACA ATT AGA TTT AGA TCA \\
\hline & & G-OR & GTG GAA TTG CTT TTT CTT CAA CAC TAA \\
\hline
\end{tabular}


universal fue: desnaturalización inicial: $95{ }^{\circ} \mathrm{C}$ por 5 minutos, seguido por 25 ciclos a $94{ }^{\circ} \mathrm{C}$ por 1 minuto, $58{ }^{\circ} \mathrm{C}$ por 2 minutos, $72{ }^{\circ} \mathrm{C}$ por 2 minutos; luego un paso de hibridación final a $58{ }^{\circ} \mathrm{C}$ por 2 minutos, una extensión final a $72{ }^{\circ} \mathrm{C}$ por 5 minutos y 5 minutos a $25^{\circ} \mathrm{C}$.

Posteriormente, se realizó una PCR anidada para detectar la familia de alelos presente en cada muestra y el polimorfismo de tamaño de los fragmentos amplificados; para el gen $m s p-1$ se analizó el bloque 2 y para el gen $m s p-2$ el bloque 3. El gen glurp no presenta familias de alelos y su análisis se hizo evaluando el polimorfismo de tamaño presente en la región $\mathrm{R} 2$.

En esta PCR la detección de cada familia se hizo por separado, es decir, un tubo para cada una con un volumen final de $20 \mu \mathrm{l}$. Las condiciones de la mezcla de reacción fueron: solución tampón 1X, $\mathrm{MgCl}_{2} 1,5 \mathrm{mM}$, dNTPs $125 \mu \mathrm{M}$, pareja de iniciadores que identifica cada alelo de msp1 (K1, MAD20 y RO33), de msp2 (IC y FC) y de glurp $125 \mathrm{nM}$ y Taq polimerasa $0,4 \mathrm{U} / \mathrm{PCR}$ y $1 \mu \mathrm{l}$ de ADN proveniente del producto de la primera PCR (PCR múltiple).

Las condiciones de amplificación para las familias de alelos de msp1 y msp2 fueron: desnaturalización inicial: $95{ }^{\circ} \mathrm{C}$ por 5 minutos, seguido por 30 ciclos así: $61{ }^{\circ} \mathrm{C}$ por 2 minutos, $72{ }^{\circ} \mathrm{C}$ por 2 minutos, 94 ${ }^{\circ} \mathrm{C}$ por 1 minuto. Luego, una hibridación final a $61{ }^{\circ} \mathrm{C}$ por 2 minutos, una extensión final a $72{ }^{\circ} \mathrm{C}$ por 5 minutos y, finalmente, 5 minutos a $25^{\circ} \mathrm{C}$. El programa para detectar los polimorfismos en glurp fue: desnaturalización inicial: $95{ }^{\circ} \mathrm{C}$ por 5 minutos, seguido por 30 ciclos a $58{ }^{\circ} \mathrm{C}$ por 2 minutos, 72 ${ }^{\circ} \mathrm{C}$ por 2 minutos y $94{ }^{\circ} \mathrm{C}$ por 1 minuto. Luego, una hibridación final a $58{ }^{\circ} \mathrm{C}$ por 2 minutos, una extensión final a $72{ }^{\circ} \mathrm{C}$ por 5 minutos y, finalmente, 5 minutos a $25^{\circ} \mathrm{C}(25-27)$.

Cada reacción de amplificación tuvo su respectivo control positivo que consistió en una mezcla de ADN de cepas de referencia que amplifican una sola familia de cada gen. HAITI amplifica la familia de alelos K1, FCB1 amplifica MAD20, 7 G8 amplifica RO33 de msp1 y la familia IC de msp2, y HB3 amplifica FC de msp2. Todas las cepas amplifican glurp. Como control negativo se usó agua.

Vale la pena mencionar que las muestras de La Carpa y Casuarito habían sido previamente genotipificadas con el gen msp1 (28). En el presente estudio estas 20 muestras fueron analizadas para los genes msp2 y glurp y con las 61 muestras restantes y las tres muestras obtenidas de los pacientes que presentaron fracaso al tratamiento, se estudiaron los tres genes.

\section{Análisis de los productos de PCR}

Para visualizar los productos de PCR de los genes $m s p 1$ y $m s p 2$ se usaron geles de agarosa al $2 \%$ y para los productos de glurp, geles de agarosa al $1,5 \%$. Los geles se tiñeron con bromuro de etidio $(1 \mu \mathrm{g} / \mathrm{ml})$ y se visualizaron bajo luz ultravioleta; el tamaño de los productos se determinó usando un patrón de peso molecular de 100 pb (Promega $\left.{ }^{\circledR}\right)$. Los resultados se registraron en el analizador de imágenes Gel Doc XR® usando el software Quality one, versión 4.6.2 para Windows ${ }^{\circledR}$ de Bio-Rad $\AA$.

\section{Aspectos éticos}

Para todos los procedimientos se tuvieron en cuenta las consideraciones éticas y los principios de la declaración de Helsinki (29), las normas del Ministerio de Salud de Colombia, Resolución 008430 de 1993 (30), y el Decreto 2737 de 1989, Artículo 13 (31).

Se elaboró de acuerdo con lo estipulado en esta resolución, un documento que fue entregado, previa lectura y explicación de la naturaleza, beneficios y riesgos de la participación en los estudios, a cada uno de los pacientes elegibles o a sus padres en caso de ser menores de edad, y un formato en el que el paciente elegible o su padre o adulto confirmó, mediante su firma, su disposición de participar.

A los pacientes elegibles cuya edad era de entre $7 y$ 18 años se les solicitó además su asentimiento. De acuerdo con la Resolución 008430, este estudio se consideró como de riesgo mínimo. Los proyectos fueron sometidos a estudio y aprobados en todas sus partes por el Comité de Ética del Instituto Nacional de Salud. Además, fueron presentados, discutidos y aprobados por las autoridades de salud de los departamentos en donde se realizaron.

\section{Resultados}

Con las 81 muestras se determinó el polimorfismo de tamaño en el gen glurp y se identificó la familia alélica presente en el gen msp2. La detección del alelo en el gen msp1 sólo se hizo con las muestras de Tierralta e Inírida, debido a que las muestras de La Carpa y Casuarito ya habían sido estudiadas previamente para este gen (28).

\section{Genotipificación de la región repetida $\mathbf{R} 2$ del gen glurp}

Se detectaron productos de PCR en un rango de tamaño de 633 a 1.009 pb. Los productos se 


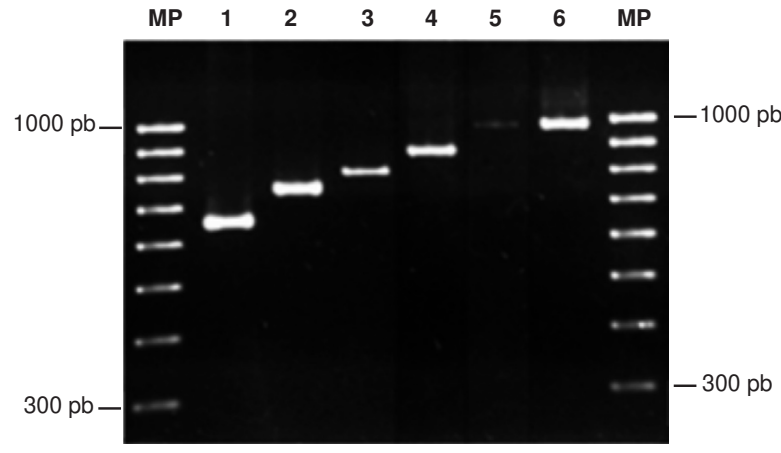

Figura 1. Genotipos encontrados en el gen glurp de Plasmodium falciparum en muestras de sangre recolectadas en cuatro localidades colombianas.

MP: marcador de 100pb; carril 1: genotipo I (rango, 600-699 pb); carril 2: genotipo II (rango, 700-799 pb); carril 3: genotipo III (rango, 800-899 pb); carril 4: genotipo IV (rango, 900-999 pb); carriles 5 y 6: genotipo $\mathrm{V}$ (rango, 1.000-1.099 pb).

clasificaron en cinco genotipos con base en el polimorfismo de tamaño encontrado así: genotipo I (600-699 pb), genotipo II (700-799 pb), genotipo III (800-899 pb), genotipo IV (900-999 pb) y genotipo V (1000-1099 pb) (figura 1). El 72,8\% de las muestras analizadas presentó el genotipo III, seguido por el genotipo II $(19,8 \%)$ y el $\mathrm{V}(3,7 \%)$. Los genotipos menos frecuentes fueron el I $(2,5 \%)$ $y$ el IV $(1,2 \%)$.

Al hacer el análisis por localidades se observó la siguiente distribución: en Tierralta, se presentó en mayor porcentaje el genotipo III, 69,5\% (IC95\% $49,9-94,3)$ y los otros genotipos se distribuyeron así: el genotipo I, 3,4\% (IC 95\% 0,4- 12,2), el II, $23,7 \%$ (IC95\% 12,9-39,8), el IV, 1,7\% (IC95\% 0,04$9,4)$, y el $\mathrm{V}, 1,7 \%$ (IC95\% 0,04-9,4). En Inírida, las dos muestras analizadas presentaron el genotipo III. En el corregimiento de La Carpa, el $80 \%$ de las muestras tuvieron el genotipo III y un $20 \%$ el genotipo II, y en Casuarito, el $80 \%$ de las muestras presentaron el genotipo III y el $20 \%$ restante el V (figura 2).

\section{Genotipificación del bloque 3 del gen msp2}

La familia de alelos IC se detectó en el 96,3\% (78/81) de las muestras, mientras que sólo cuatro muestras $(4,9 \%)$, dos procedentes de Tierralta y dos de Casuarito, presentaron la familia FC. En todo el estudio sólo se encontró una infección mixta que correspondió a una muestra de Casuarito, en la cual se observó amplificación tanto para la familia IC como para la FC. Se detectaron bandas de diferentes tamaños en ambas familias, pero no se hizo un análisis más fino para determinar con

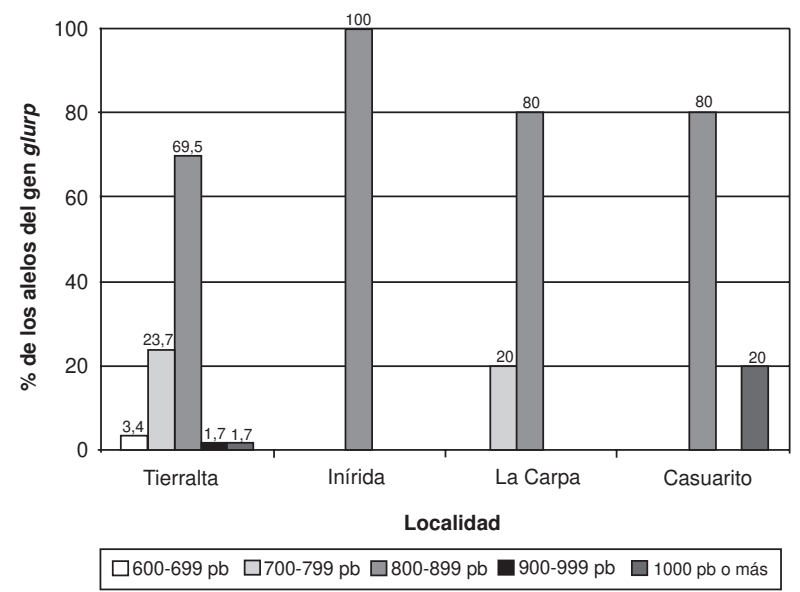

Figura 2. Genotipos detectados para el gen glurp en las cuatro localidades.

exactitud su tamaño y establecer variantes (por tamaño) dentro de cada familia de alelos.

\section{Genotipificación del bloque 2 del gen msp1}

El alotipo MAD20 se detectó en el $100 \%$ de las muestras (59 de Tierralta y 2 de Inírida) y todas presentaron una banda de $187 \mathrm{pb}$. No se encontraron los alotipos $\mathrm{K} 1$ o RO33.

\section{Glurp en la diferenciación de infecciones nuevas y recurrentes}

Entre los pacientes de Tierralta que fueron incluidos en el estudio de eficacia, uno presentó fracaso terapéutico precoz y dos presentaron fracaso terapéutico tardío (24). Las muestras pareadas (día 0 y día de la falla) de estos tres pacientes fueron genotipificadas con el gen glurp y mostraron el mismo patrón de tamaño, lo que confirmó que estos pacientes habían presentado recrudecimientos (figura 3 ).

Por otra parte, en la misma población se presentó un paciente que ingresó dos veces al estudio, la segunda, tres meses después de la primera, y presentó una respuesta clínica adecuada en las dos. Se analizaron las muestras del día 0 de ambas ocasiones y se detectaron patrones de tamaño distintos, lo cual sugiere que este paciente sufrió una nueva infección (figura 3).

\section{Discusión}

Los resultados permiten concluir que con la metodología utilizada, PCR y visualización de amplicones en geles de agarosa, de los tres genes usados para genotipificar $P$. falciparum, el 


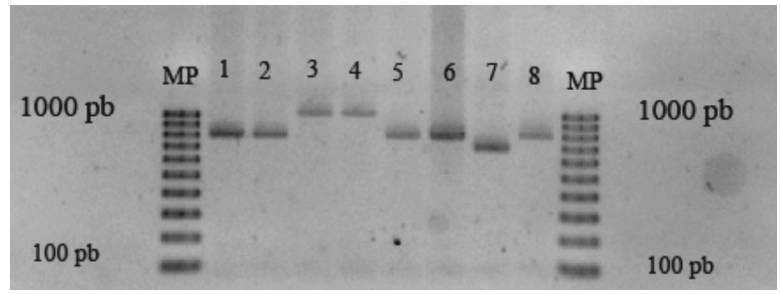

Figura 3. Genotipos encontrados en el gen glurp en tres pacientes con recrudecimiento de la infección palúdica y en un paciente con reinfección.

MP: marcador de peso molecular de $100 \mathrm{pb}$; carril 1: muestra del paciente 10 en el día 0; carril 2: muestra del paciente 10 en el día del fracaso terapéutico (día 28); carril 3: muestra del paciente 12 en el día 0; carril 4: muestra del paciente 12 en el día del fracaso terapéutico (día 2); carril 5: muestra del paciente 21 en el día 0; carril 6: muestra del paciente 21 en el día del fracaso terapéutico (día 28); carril 7: muestra del día 0 de la primera infección del paciente que ingresó dos veces al estudio; carril 8: muestra del día 0 de la segunda infección del paciente anterior.

gen glurp fue el marcador molecular que brindó mayor información sobre la composición de las poblaciones naturales de $P$. falciparum en las muestras analizadas. Lo anterior se pudo evidenciar con los resultados de la localidad de Tierralta, donde se encontraron cinco genotipos distintos, lo cual refleja la diversidad genética de los parásitos que circulan en esta zona del país. Esta interpretación no podría hacerse con el resultado obtenido para el gen $m s p 1$, ya que en Tierralta sólo circula la familia MAD20 con un tamaño de $187 \mathrm{pb}$.

En el caso del gen msp2, la familia de alelos IC predominó, no sólo en Tierralta sino en las otras tres localidades, y la familia de alelos FC solamente se observó en cuatro muestras, dos de Tierralta y dos de Casuarito. En ambas familias se evidenció polimorfismo de tamaño, y se encontraron bandas en un rango entre 467 y 513 pb para IC y entre 286 y 300 pb para FC. Teniendo en cuenta que la familia predominante es la IC y que el rango de tamaño es más amplio, este gen puede ser de utilidad en los estudios de diversidad genética si se logran establecer diferencias en el número de pares de bases entre las bandas obtenidas en la PCR. Lo anterior plantea la necesidad de usar metodologías más finas, como el uso de geles de poliacrilamida, para establecer los tamaños de las bandas y establecer claramente el polimorfismo de tamaño.

En relación con el gen msp1, nuestros resultados al igual que los de otros investigadores $(12,20,28)$ muestran el predominio de la familia alélica MAD20. No se detectó polimorfismo de tamaño, y se encontró en todas las muestras de Tierralta e Inírida una banda de 187 pb. Los resultados de Guerra et al. (28) mostraron que en La Carpa sólo se encontró la familia alélica MAD20, mientras que en Casuarito se detectaron las tres familias; sin embargo, al igual que en este estudio, no se observó polimorfismo de tamaño en MAD20 y siempre se obtuvo una banda de $150 \mathrm{pb}$.

Vale la pena mencionar que los iniciadores usados en ambos estudios fueron distintos y, por ello, las diferencias en los tamaños de los amplicones. Lo anterior sugiere que el gen $m s p 1$ no es un buen marcador para estudios de diversidad genética en Colombia y se debería dejar de evaluar, ya que los resultados no logran aportar información que conlleve a distinguir un genotipo parasitario de otro. Este comportamiento es totalmente diferente al que ocurre en algunas regiones de África y del sureste asiático, donde se ha encontrado un alto número de variantes para $m s p 1, m s p 2$ y glurp $(14,21,32-34)$.

Si bien en las cuatro localidades estudiadas se observó polimorfismo de tamaño en el gen glurp, se destaca Tierralta por el mayor número de genotipos encontrados. Aunque esto podría explicarse por el mayor número de muestras estudiadas procedentes de Tierralta, también puede estar relacionado con la alta incidencia de malaria en la zona, que se refleja en un índice parasitario anual para el año 2006 de 202 por 1.000 habitantes, comparado con las otras tres localidades: Inírida, que presentó un índice parasitario anual para el año 2007 de 12 por 1.000 habitantes, San José del Guaviare, de 51 por 1.000 habitantes, y Puerto Carreño, de 40 por 1.000 habitantes durante el año 2004; los dos índices parasitarios anuales anteriores corresponden a los datos por municipio y no por corregimiento, ya que no se dispone de esa información (comunicación personal, Subdirección de Vigilancia, Instituto Nacional de Salud). Por otro lado, teniendo en cuenta las diferencias entre el número de muestras de cada localidad, somos conscientes de la necesidad de realizar nuevos estudios en estas zonas, usando métodos apropiados de muestreo y mayores tamaños de muestra.

Vale la pena resaltar que, de los tres genes empleados en este estudio, sólo glurp permitió identificar pacientes con infección recurrente o con reinfección. En el primer caso logró determinar la presencia del mismo parásito tanto en el día 0 como en el día de la falla y en el segundo caso pudo diferenciar los parásitos recolectados en el 
día 0 en un paciente que ingresó dos veces al estudio. En este último caso, los resultados para el gen $m s p 1$ y $m s p 2$ dieron exactamente igual en ambos días MAD20 e IC, respectivamente.

A pesar de los pocos estudios que se han realizado en Colombia con el gen glurp $(12,22,35)$, los resultados a la fecha han mostrado que este gen aporta mayor información sobre la diversidad genética de $P$. falciparum que los datos obtenidos con msp1 y msp2. Un estudio realizado en Antioquia en los municipios de Turbo y Zaragoza, entre marzo y junio del 2000, reportó cuatro genotipos con bandas entre 800 y 900 pb (12), otro en Quibdó, efectuado entre marzo y julio de 2001, reportó tres variantes con pesos entre 650 y $1.100 \mathrm{pb}$ (22) y otro realizado con muestras de 10 localidades ubicadas en distintas regiones de Colombia, recolectadas entre noviembre de 2002 y junio de 2003, mostró 11 variantes alélicas distintas. En Suramérica existen algunos trabajos que han utilizado este marcador: en Surinam se reportaron dos genotipos (600 pb, 700 pb) (36), al igual que en Venezuela (883 pb, $1.000 \mathrm{pb)} \mathrm{(11);}$ en Perú, entre 1998 y 1999, se reportó un estudio con ocho genotipos que van desde 450 hasta 800 pb (13). En Centroamérica, específicamente en Honduras, se reportaron dos genotipos para glurp, uno de $575 \mathrm{pb}$ y otro de $700 \mathrm{pb}$ (37).

En el resto del mundo se pueden citar investigaciones desarrolladas en varios países, como en Tailandia en 1995, donde reportaron siete genotipos distintos que van en un rango de 800 a 1.200 pb y, en 1999, donde se reportaron 12 genotipos entre 660 y 1.100 pb $(9,38)$. En Sudán se reportaron 16 a 21 genotipos, en Nigeria 5 genotipos en un rango de 225 a 900 pb y, en un estudio donde se incluyeron pacientes de África, Tailandia y Surinam, se evidenciaron 5 genotipos comunes que estaban en el rango de 300 a 800 pb (39-42).

En la actualidad, en Colombia se han venido realizando investigaciones en las cuales se usan otros marcadores llamados microsatélites, aunque no se ha dejado claro cuál microsatélite es el más apropiado para este tipo de estudios, se ha sugerido que con sólo uno de ellos es suficiente para obtener información valiosa sobre el polimorfismo genético del parásito $(34,42)$.

Las evidencias aportadas por este estudio en cuanto a la diversidad genética encontrada en las cuatro localidades estudiadas, mediante métodos de genotipificación convencional, muestran la importancia de utilizar el gen glurp, ya que brinda información valiosa pues permite detectarfácilmente variaciones entre las poblaciones de $P$. falciparum que circulan en el país. El empleo del gen glurp puede, además, ser de gran utilidad en estudios de eficacia, ya que permite establecer diferencias entre clones de parásitos. Así, podría ayudar a distinguir pacientes que sufren recrudecimiento de la infección o reinfección, aspecto de gran importancia en los estudios de eficacia. Sin embargo, debido al escaso número de pacientes con reinfecciones o recrudecimiento de la infección, es necesario realizar estudios adicionales, en los que se pueda analizar un mayor número de muestras que permitan confirmar estos hallazgos.

\section{Agradecimientos}

Los autores agradecen la colaboración recibida de las siguientes instituciones para la realización del trabajo de campo: Secretaría Departamental de Salud de Córdoba, Secretaría Municipal de Salud de Tierralta; microscopistas de la empresa asociativa de trabajo DIFUSANJOR, Secretaría Departamental de Salud de Guaviare, Puesto de Salud de La Carpa, Secretaría Departamental de Salud del Vichada, Laboratorio Departamental de Salud Pública de Vichada y Puesto de Salud de Casuarito.

\section{Conflicto de intereses}

Los autores manifestamos expresamente que durante la realización del presente trabajo no existió conflicto de interés alguno que pudiera haber afectado los resultados obtenidos.

\section{Financiación}

Este estudio fue cofinanciado con recursos del Fondo de Investigaciones en Salud, Ministerio de la Protección Social-Colciencias, proyecto código 2104-04-16338, contrato No. RC 363-2004, del Instituto Nacional de Salud y de la Secretaría Departamental de Desarrollo de la Salud de Córdoba.

\section{Referencias}

1. World Health Organization, UNICEF, Roll Back Malaria. World Malaria Report 2006. Fecha de consulta: 8 de mayo de 2007. Disponible en: http://www.rbm.who.int/wmr2006/ index.html.

2. Ministerio de la Protección Social, Instituto Nacional de Salud, Subdirección de Vigilancia y Control en Salud Pública. Sistema de Vigilancia en Salud Pública (SIVIGILA). Informe final de malaria. Semanas 1-52. Bogotá: Ministerio de la Protección Social, INS; 2007. Fecha de consulta: 8 de mayo de 2008. Disponible en: http://www.ins.gov. $\mathrm{co} /$ ? idcategoria $=1731 \& \mathrm{pag}=3$. 
3. Yongyuth Y. Basis for antifolate action and resistance in malaria. Microbes Infect. 2002;4:175-82.

4. Ferreira MU, Ribeiro WL, Tonon AP, Kawamoto F, Rich S. Sequence diversity and evolution of the malaria vaccine candidate merozoite surface protein-1 (MSP-1) of Plasmodium falciparum. Gene. 2003;304:65-75.

5. Miller L, Roberts T, Shahanbuddin M, McCutcham T. Analysis of sequence diversity in the Plasmodium falciparum merozoite surface protein-1 (MSP-1). Mol Biochem Parasitol. 1993;59:1-14.

6. Fenton B, Clark J, Khan A, Robinson J, Walliker D, Ridley R, et al. Structural and antigenic polymorphism of the 35- to 48- kilodalton merozoite surface antigen (MSA-2) of the malaria parasite Plasmodium falciparum. Mol Cell Biol. 1991;11:963-71.

7. Borre M, Dziegiel M, Hogh B, Petersen E, Rieneck $\mathrm{K}$, Riley E, et al. Primary structure and localization of a conserved immunogenic Plasmodium falciparum glutamate rich protein (GLURP) expressed in both the preerythrocytic and erytrocytic stages of the vertebrate life cycle. Mol Biochem Parasitol. 1991;49:119-31.

8. Roper C, Elhassan I, Hviid L, Giha H, Richardson W, Babiker $\mathbf{H}$, et al. Detection of very low level Plasmodium falciparum infections using the nested polymerase chain reaction and a reassessment of the epidemiology of unstable malaria in Sudan. Am J Trop Med Hyg. 1996;54:325-31.

9. Viriyakosol S, Siripoon N, Petcharapirat C, Percharapirat P, Jarra W, Thaithong S, et al. Genotyping of Plasmodium falciparum isolates by the polymerase chain reaction and potential uses in epidemiological studies. Bull World Health Organ. 1995;73:85-95.

10. Dorsey G, Gasasira A, Machekano R, Kamya M, Staedke $\mathbf{S}$, Hubbard A. The impact of age, temperature, and parasite density on treatment outcomes from antimalarial clinical trials in Kampala, Uganda. Am J Trop Med Hyg. 2004;71:531-6.

11. Tami A, Grundmann A, Sutherland C, Mcbride J, Cavanagh DR, Campos E, et al. Restricted genetic and antigenic diversity of Plasmodium falciparum under mesoendemic transmission in the Venezuelan Amazon. Parasitology. 2002;124:569-81.

12. Montoya L, Maestre A, Carmona J, Lopes D, do Rosario VE, Blair S. Plasmodium falciparum: diversity studies of isolates from two Colombian regions with different endemicity. Exp Parasitol. 2003;104:14-9.

13. Hijar G, Quino H, Padilla C, Montoya Y. Variabilidad genética de Plasmodium falciparum en pacientes con malaria grave y malaria no complicada en lquitos, Perú. Rev Peru Med Exp Salud Pública. 2002;19:131-5.

14. Muguttu K, Priotto G, Guthmann J, Kigali J, Adjuik M, Snounou G, et al. Molecular genotyping in malaria treatment trial in Uganda unexpected high rate of new infections within 2 weeks after treatment. Trop Med Int Health. 2007;12:219-23.

15. Jongwutiwes S, Tanabe K, Nakazawa S, Yanagi T, Kanbara $\mathbf{H}$. Sequence variation in the tripeptide repeats and $\mathrm{T}$ cell epitopes in P190 (MSA-1) of Plasmodium falciparum from field isolates. Mol Biochem Parasitol. 1992;51:81-90.
16. Raj D, Bibhu R, Dash A, Supakar P. Genetic diversity in the merozoite surface protein 1 gene of Plasmodium falciparum in different malaria endemic localities. Am J Trop Med Hyg. 2004;71:285-9.

17. Marshall VM, Coppel RL, Anders R, Kemp D. Two novel alleles within subfamilies of the merozoite surface antigen 2 (MSA-2) of Plasmodium falciparum. Mol Biochem Parasitol. 1992;50:181-4.

18. Snewin V, Herrera M, Sánchez G, Scherf A, Langsley G, Herrera S. Polymorphism of the alleles of the merozoite surface antigens MSA1 and MSA2 in Plasmodium falciparum wild isolates from Colombia. Mol Biochem Parasitol. 1991;49:265-76.

19. Terrientes Z, Vergara J, Kramer K, Herrera S, Chang S. Restricted genetic diversity of Plasmodium falciparum major merozoite surface protein 1 in isolates from Colombia. Am J Trop Med Hyg. 2005;73(Suppl.5):55-61.

20. Gómez D, Chaparro J, Rubiano C, Rojas O, Wasserman M. Genetic diversity of Plasmodium falciparum field samples from an isolated Colombia village. Am J Trop Med Hyg. 2002;67:611-6.

21. Guerra-Neira A, Rubio JM, Royo JR, Ortega JC, Auñón AS, Díaz PB, et al. Plasmodium diversity in non-malaria individuals from the Bioko Island in Equatorial Guinea (West Central Africa). Int J Health Geogr. 2006;5:27.

22. Pardo L, González IJ, Osorio L. Determinación de la multiplicidad de infección de Plasmodium falciparum por medio de la amplificación por PCR de los genes msp1, msp2 y glurp en Quibdó, Chocó. 2001. CIDEIM. p. 12-47. Fecha de consulta: 27 de julio de 2008. Disponible en: http://www.scribd.com/doc/3227258/Vol623-g

23. Montoya L, Maestre A, Blair S, Carmona J. Variabilidad genética en cepas de Plasmodium falciparum circulantes en regiones colombianas con riesgo diferente de malaria. Fecha de consulta: 27 de julio de 2008. Disponible en: http://www. ces.edu.co/Descargas\%5CPubl_Med_Vol15_2\%5C4995_15-2.pdf.

24. Pérez MA, Cortés LJ, Guerra AP, Knudson A, Usta C, Nicholls RS. Eficacia de la combinación amodiaquina más sulfadoxina-pirimetamina y de la cloroquina para el tratamiento de paludismo en Córdoba, Colombia, 2006. Biomédica. 2008;28:148-59.

25. Snounou G, Beck HP. The use of PCR genotyping in the assessment of recrudescence or reinfection after antimalarial drug treatment. Parasitol Today. 1998; 14:462-7.

26. Kho WG, Chung JY, Sim EJ, Kim MY, Kim DW, Jongwutiwes $\mathrm{S}$, et al. A multiplex polymerase chain reaction for a differential diagnosis of Plasmodiun falciparum and Plasmodiun vivax. Parasitol Int. 2003;52:229-36.

27. Rubio JM, Roche PJ, Moyano E, Benito A. The potential utility of the semi-nested multiplex PCR technique for the diagnosis and investigation of congenital malaria. Diagn Microbiol Infect Dis. 2000;38:233-6.

28. Guerra AP, Knudson A, Nicholls RS, Galindo J, Ravid Z, Rahirant S, et al. Genotipificación de los genes msp1 (bloque 2) y dhfr (codón 108) de Plasmodium falciparum en muestras de campo recolectadas en cuatro localidades endémicas de Colombia. Biomédica. 2006;26:101-12. 
29. World Medical Association. Declaration of Helsinki. Ethical principles for medical research involving human subjects. Last revision. 52 ${ }^{\text {nd }}$ WMA General Assembly. Edinburgh (Scotland): World Medical Association; 2000.

30. Ministerio de Salud. Dirección de desarrollo científico y tecnológico. Normas científicas, técnicas y administrativas para la investigación en salud. Resolución No. 008430 de 1993. Santafé de Bogotá: Imprenta Nacional; 1993.

31. Congreso de la República de Colombia. Código del menor. Derechos del niño. Decreto 2737, artículo 13. Bogotá D.C.: Congreso de la República de Colombia; 1989.

32. Cattamanchi A, Kyabayinze D, Hubbard A, Rosenthal P, Dorsey G. Distinguishing recrudescence from reinfection in a longitudinal antimalarial drug efficacy study: comparison of results based on genotyping of $m s p 1, m s p 2$ and glurp. Am J Trop Med Hyg. 2003;68:133-9.

33. Slater M, Kiggundu M, Dokomajilar C, Kamya M, Bakyaita $\mathbf{N}$, Talisuna A, et al. Distinguishing recrudescences from new infections in antimalarial clinical trials: major impact of interpretation of genotyping results on estimates of drug efficacy. Am J Trop Med Hyg. 2005;73:256-62.

34. Mugittu K, Adjuik M, Snounou G, Ntoumi F, Taylor W, Mshinda $\mathbf{H}$, et al. Molecular genotyping to distinguish between recrudescent and new infections in treatment trials of Plasmodium falciparum malaria conducted in SubSaharan Africa: Adjustment of parasitological outcomes and assessment of genotyping effectiveness. Trop Med Int Health. 2006;11:1350-9.

35. Jiménez JN, Snounou G, Letourneur F, Rénia L, Vélez ID, Muskus CE. Near-fixation of a Pfmsp1 block 2 allelic variant in genetically diverse Plasmodium falciparum populations across Western Colombia. Acta Trop. 2010;114:67-70.36.
36. Peek R, Gool TV, Panchoe D, Greve S, Bus E, Resida L. Drug resistance and genetic diversity of Plasmodiun falciparum parasites from Suriname. Am J Trop Med Hyg. 2005;73:833-8.

37. Haddad D, Snounou G, Mattei D, Enamorado I, Figueroa J, Stahl S, et al. Limited genetic diversity of Plasmodium falciparum in field isolates from Honduras. Am J Trop Med Hyg. 1999;60:30-4.

38. Snounou G, Xinping Z, Siripoon N, Jarra W, Viriyakosol S. Biased distribution of $m s p 1$ and $m s p 2$ allelic variants in Plasmodium falciparum populations in Thailand. Trans $\mathrm{R}$ Soc Trop Med Hyg. 1999;93:369-74.

39. Babiker HA, Abdel AA, Hamad A, Mackinnon MJ. Population dynamics of Plasmodium falciparum in an unstable malaria area of Sudan. Parasitology. 2000;120:10511.

40. Happi C, Gbotosho G, Sowunmi A, Falade C, Akinboye D, Gerena L, et al. Molecular analysis of Plasmodium falciparum recrudescent malaria infections in children treated with chloroquine in Nigeria. Am J Trop Med Hyg. 2004;70:20-6.

41. Farnert A, Bjorkman A. Short report: limited advantage of multiple consecutive samples for genotyping Plasmodium falciparum populations during the first days of treatment. Am J Trop Med Hyg. 2005;73:204-6.

42. Mlambo G, Sullivan D, Mutambu S, Soko W, Mbedzi J, Chivenga $\mathrm{J}$, et al. Analysis of genetic polymorphism in select vaccine candidate antigens and microsatellite loci in Plasmodium falciparum from endemic areas at varying altitudes. Acta Trop. 2007;102:201-5. 